\title{
Micro-Displace Sensor Based on Self-Mixing Interference of the Fiber Laser With Phase Modulation
}

\author{
Hui HAO*, Dongmei GUO, Ming WANG, Wei XIA, and Xiaoqi NI \\ Jiangsu Key Lab of Opto-Electronic Technology, School of Physical Science and Technology, Nanjing Normal \\ University, Nanjing, 210023, China \\ *Corresponding author: Hui HAO E-mail:huihao@njnu.edu.cn
}

\begin{abstract}
Micro-displacement measurement based on self-mixing interference using a fiber laser system was demonstrated. The sinusoidal phase modulation technique was introduced into the fiber laser self-mixing interference measurement system to improve the measurement resolution. The phase could be demodulated by the Fourier analysis method. Error sources were evaluated in detail, and the system was experimentally applied to reconstruct the motion of a high-precision commercial piezoelectric ceramic transducer (PZT). The displacement measurement resolution was well beyond a half-wavelength. It provides a practical solution for displacement measurement based on all optical-fiber sensing applications with high precision.
\end{abstract}

Keywords: Self-mixing interference, micro-displacement sensing, phase modulation, fiber laser

Citation: Hui HAO, Dongmei GUO, Ming WANG, Wei XIA, and Xiaoqi NI, "Micro-Displace Sensor Based on Self-Mixing Interference of the Fiber Laser With Phase Modulation," Photonic Sensors, 2014, 4(4): 379-384.

\section{Introduction}

In recent decades, with the steadily increasing importance of micro-electro-mechanical system (MEMS) structures in different technology fields, it becomes more crucial to measure the displacement of mechanical components with a high accuracy of a few nanometers [1-4]. The optical measurement techniques including the Michelson interferometer, Fabry-Perot (F-P) interferometer, and heterodyne interferometer, etc. are of great interest and importance because they are fast, contamination free, non-destructive and have a good in-line capability [5]. Self-mixing interference (SMI) occurs when a portion of light emitted from the laser is reflected or backscattered by an external object and is allowed to re-enter the laser cavity, causing the variation of both the output power and spectra of the laser [6].
Compared with the well-established interferometers, the self-mixing interferometer is characterized by its capability of high performance with an extremely simple and inexpensive experimental setup and the potential for direct industrial applications for vibration and displacement sensing [7, 8]. In recent years, the active sensing applications based on fiber laser are attracting attentions of the researchers. The researches using the self-mixing interference of a fiber laser for velocity sensing [9], remote measurement application [10], and vibration measurement [11] have been reported. Fiber laser SMI is used to measure the displacement with an accuracy of $\lambda / 2$ by counting interference signal peaks [12]. In order to increase the accuracy beyond $\lambda / 2$, some methods for the analysis of the fiber SMI signal have been reported, and an accuracy of the 
$\lambda / 10$ could be obtained [13]. Generally, the precision does not meet the demand of the high-accuracy measurement today.

The sinusoidal phase modulation in the interferometer has played a very important role to achieve a high precision in displacement measurement $[14,15]$. In order to further improve the measurement resolution, the phase modulation technique is introduced into the active fiber measurement system. The phase modulation is realized by a high-speed fiber stretcher, which provides a good signal to noise ratio (SNR), low insertion loss, and high modulation frequency. The displacement measurement resolution is well beyond a half-wavelength.

\section{Manuscript principle}

Assuming that SMI operates in a low-feedback regime where the effect of multiple reflections in the external cavity is small, the interference signal detected by the photodetector (PD) with sinusoidal phase modulation $\varphi(t)$ is given by [15-17]

$$
I(t)=I_{0}\left\{1+m \cos \left[\phi(t)+a \sin \left(2 \pi f_{m} t+\beta\right)\right]\right\}
$$

with

$$
\phi(t)=4 \pi n L(t) / \lambda_{0}
$$

where $I_{0}$ denotes the unperturbed optical output power, $\phi(t)$ is the measured phase of the external cavity, $m$ is the fringe visibility, $n$ is the refractive index of air, $L(t)$ is the length of the external cavity, and $\lambda_{0}$ is the central wavelength in vacuum. $\varphi(t)=a \sin \left(2 \pi f_{m} t+\beta\right) \quad$ is the sinusoidal phase modulation term, where $a$ is the modulation depth, $f_{m}$ is the modulation frequency, and $\beta$ is the initial phase. Expanding (1), we obtain

$$
\begin{aligned}
& I(t)=I_{0}+m I_{0} \cos \phi J_{0}(a) \\
& +m I_{0} \cos \phi\left[2 \sum_{n=1}^{\infty} J_{2 n}(a) \cos \left((2 n)\left(2 \pi f_{m} t+\beta\right)\right)\right] \\
& -m I_{0} \sin \phi\left[2 \sum_{n=0}^{\infty} J_{(2 n+1)}(a) \sin \left((2 n+1)\left(2 \pi f_{m} t+\beta\right)\right)\right]
\end{aligned}
$$

The interference signal can be demodulated by the Fourier analysis method. Equation (4) shows the amplitudes of harmonics at the frequencies $f_{m}$ and $2 f_{m}$ have the following expressions:

$$
\begin{gathered}
I\left(f_{m}, t\right)=-2 m I_{0} \sin \phi(t) J_{1}(a) \sin \left(2 \pi f_{m} t+\beta\right)= \\
A_{1}(t) \sin \left(2 \pi f_{m} t+\beta\right) \\
I\left(f_{2 m}, t\right)=2 m I_{0} \cos \phi(t) J_{2}(a) \cos \left(4 \pi f_{m} t+\beta\right)= \\
A_{2}(t) \cos \left(4 \pi f_{m} t+\beta\right)
\end{gathered}
$$

where $J_{n}(a)$ is the $n$ th-order Bessel function. The Fourier analysis method is proposed to determine the values of $A_{1}(t)$ and $A_{2}(t)$. Then, we can calculate the phase $\phi(t)$ from $A_{1}(t)$ and $A_{2}(t)$ using the subsequent relation:

$$
\phi(t)=\arctan \left[\frac{A_{1}(t)}{A_{2}(t)} * \frac{J_{2}(a)}{J_{1}(a)}\right] .
$$

The phase $\phi$ obtained using the demodulation technique proposed above is wrapped within the region of $-\pi$ and $\pi$. After a phase unwrapping process and following the relationship between the phase $\phi$ and the length of the external cavity, the displacement of the external target can be reconstructed.

\section{Simulation results and error analysis}

In order to evaluate the displacement measurement technique proposed, the simulation result is presented. Figure 1(a) shows the simulated micro-motion of the external target with the amplitude $6 \mu \mathrm{m}$ and frequency $10 \mathrm{~Hz}$. The modulation frequency is $8 \mathrm{kHz}$, and the phase shifting amplitude is chosen to be $1.22 \mathrm{rad}$ for the reasons to be described later. The wavelength is $1550 \mathrm{~nm}$, and the fringe visibility $m$ is 0.1 . Figure 1(b) shows the demodulated phase. Equation(6) is used to calculate the standard deviation of the measured displacement:

$$
\sigma=\sqrt{\sum_{i=1}^{n}\left(L_{i m}-L_{i r}\right)^{2} /(n-1)}
$$

where $L_{i m}$ is the extracted displacement at the $i$ th point, $L_{i r}$ is the actual displacement at the $i$ th point, and $n$ is the number of sampling points during the whole measurement process. The magnitude of the measurement error is about $0.51 \mathrm{~nm}$ with the standard deviation $\sigma$ of $0.13 \mathrm{~nm}$. In this paper, we consider mainly the effect of the instability of the phase shift amplitude and the multiple reflections in the external cavity. 


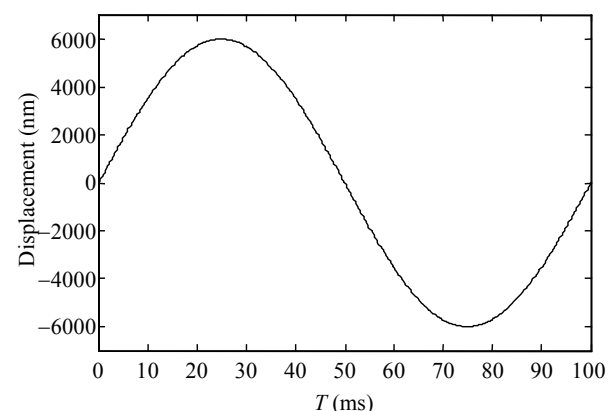

(a)

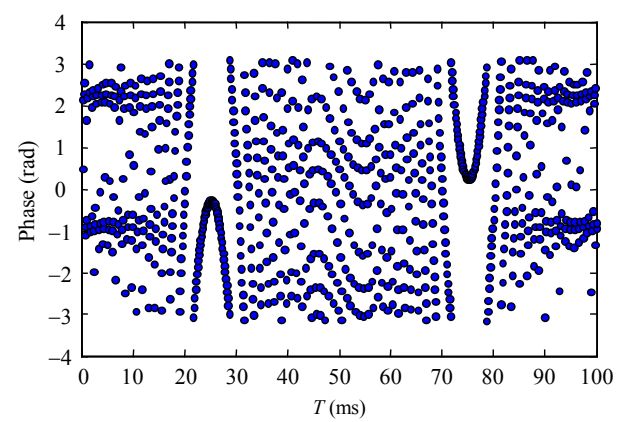

(b)

Fig. 1 Simulation results: (a) simulated displacement and (b) wrapped measured phase.

A classic performance criterion for the phaseshifting interferometer is the sensitivity to deviations in the phase shift amplitude excursion from the expected value. The random fluctuations in the phase shifting amplitude affect the accuracy in calculation of $\phi$ from (1), and the SMI signal including the instability of the phase shifting amplitude can be written as [18]

$$
I(t)=I_{0}\left\{1+m \cos \left[\phi+a \sin \left(2 \pi f_{m} t\right)+n_{a}(t)\right]\right\}
$$

where $n_{a}(t)$ is the time-dependent fluctuation of the phase shifting amplitude. It is assumed that the noise $n_{a}(\mathrm{t})$ is the Gaussian distribution with zero means and variance $\sigma_{a}^{2}$. Figure 2 shows the relationship between the standard deviation and phase shift amplitude $a$, where $a$ is regarded from $0.25 \mathrm{rad}$ to $1.75 \mathrm{rad}$ in the condition $\sigma_{a} \in(0.005 \pi, 0.02 \pi)$. The principle of the proposed interferometer is based on the consideration of one feedback from the external cavity. When the effect of multiple reflections can not be neglected completely, the emitted optical power of the laser can be written as [19]

$$
I(t)=I_{0}\left\{1+m \cos \left[\phi(t) \sum_{j=0}^{\infty}(-\eta)^{\mathrm{j}}\right] \cos [\mathrm{j} \phi(t)]\right\} .
$$

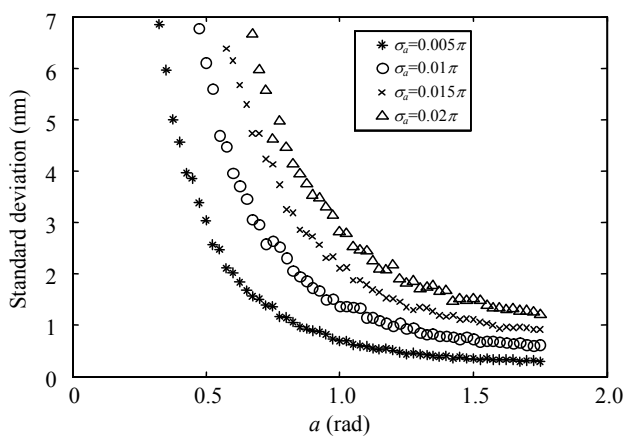

Fig. 2 Relationship between the standard deviation and $a$ with $\sigma_{a} \in(0.005 \pi, 0.02 \pi)$.

The lowest few order terms in (1) (i.e., $j=1,2, \cdots$ ) begin to act, and measurement error due to multiple reflections occurs. For the running state of the interferometer is restricted within the weak feedback regime, the multiple feedback is not dramatic. So only the lowest 6th feedback is considered, and the simulated results of standard deviation in the reconstructed displacement changing with the $n$ thorder reflections and undulation coefficient $m$ are shown in Fig. 3.

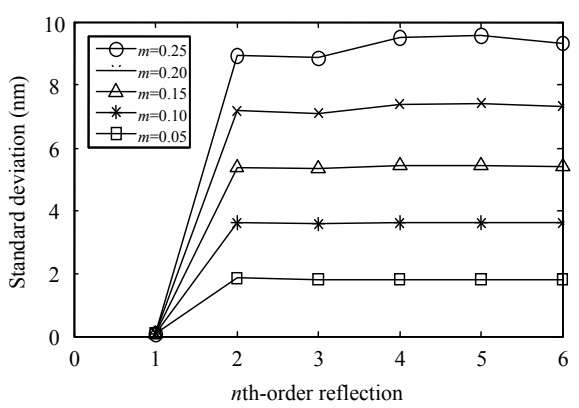

Fig. 3 Standard deviation of the reconstructed displacement due to multiple reflections.

For a certain undulation coefficient $m$, the standard deviation experiences a sharp increase if the second order reflection is taken into consideration. And an increase in the deviation is proportional to the increment of the undulation coefficient. When $m$ is small, the deviation remains almost invariant even if more high order reflections are included. However, it is obviously seen that there is a fluctuation in the deviation if $m$ is continually increased and larger than 0.15 . As a result, it is possible to reduce this measurement error at the cost of a detected SMI signal with the low undulation coefficient. We can get that the standard 
deviation derived from the multiple reflection effect is about $3.65 \mathrm{~nm}$ when $m$ is less than 0.1 . And the standard deviation is about $1.8 \mathrm{~nm}$ as $m=0.05$.

\section{Experimental results and discussion}

The experimental setup, which is illustrated in Fig. 4, could be mainly divided into 2 parts: the optical part and the electronic part. The active medium was a section of 10-m length erbium-doped fiber, and it was pumped by a $980-\mathrm{nm}$ laser diode with $140-\mathrm{mW}$ power through a wavelength division multiplexer (WDM) coupler. The bandwidth of the fiber Bragg grating (FBG) was less than $0.3 \mathrm{~nm}$, and the reflection coefficient of the FBG was $90 \%$ with a Bragg wavelength $1550 \mathrm{~nm}$. Two circulators were used to separate the emission and reception channels. This scheme ensured the isolation of the laser from the modulator and avoided parasitic feedback into the laser cavity. In addition, the optical feedback from the target suffered from the minimal losses and was directly re-injected into the laser. The phase modulation was realized by a high-speed fiber stretcher (OPTIPHASE, PZ1), which provided a good SNR, low insertion loss, and high modulation frequency. The light was reflected back to the laser cavity by a vibrating silicon wafer driven by a piezoelectric ceramic transducer (PZT) translator (PI, P-762.2L). The reentered light mixed with the original light in the laser cavity, producing self-mixing interference. The interference signal was detected by a PD, after amplified (New Focus, 1611 module) and analog-to-digital (A/D) converted (National Instrument, NI PCI-6251), and then sent to the signal processing unit.

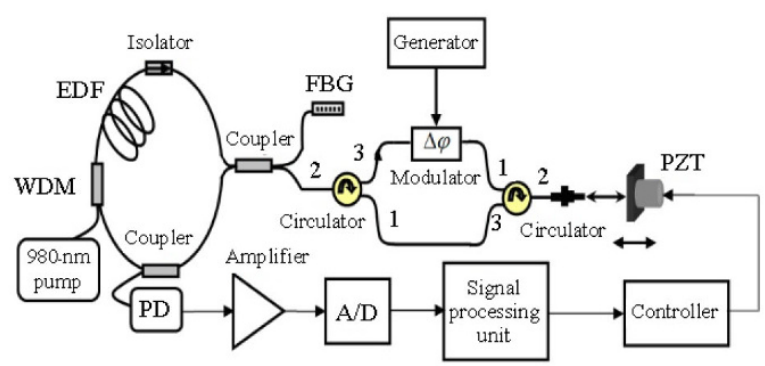

Fig. 4 Experimental setup.

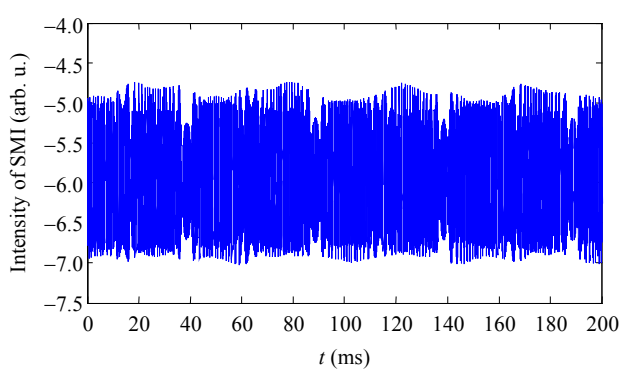

(a)

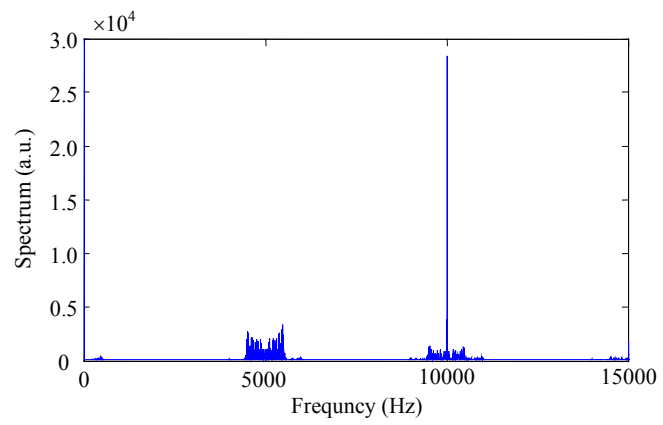

(b)

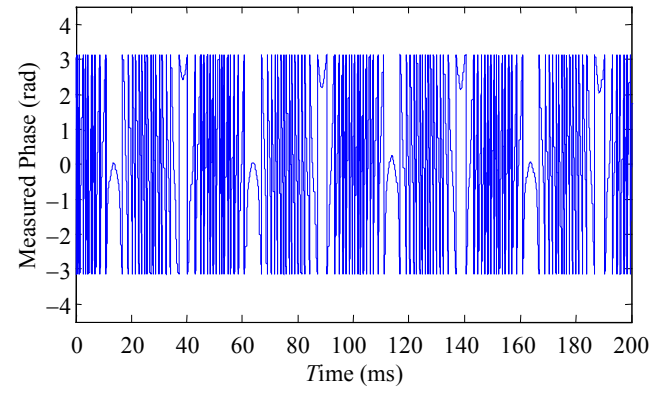

(c)

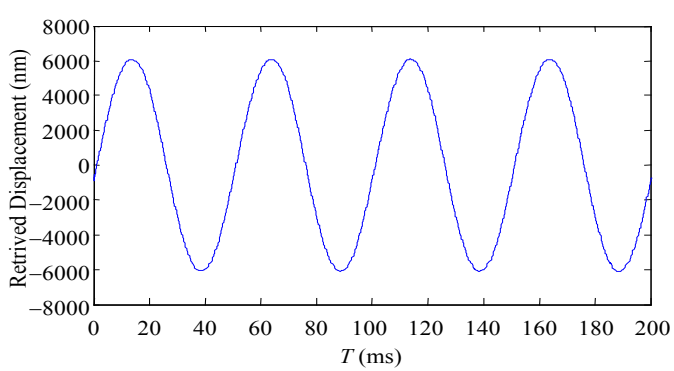

(d)

Fig. 5 Displacement measurement results with a frequency $20 \mathrm{~Hz}$, and an amplitude $6000 \mathrm{~nm}$ : (a) SMI signal, (b) frequency spectrum of the SMI signal, (c) wrapped measured phase, and (d) reconstructed displacement.

Experiments have been done to confirm the validity of the sinusoidal phase modulating self-mixing interference and the phase demodulation method. In our experiment, the first rising edge of the transistor-transistor logic (TTL) level from the signal generator which drived the modulator was 
introduced to the analog-to-digital converter card as the trigger of data acquisition. First, the PZT was controlled to move at a sinusoidal form with a frequency $20 \mathrm{~Hz}$ and an amplitude $6 \mu \mathrm{m}$. The modulation frequency was $5 \mathrm{kHz}$, and the modulation depth was $1.22 \mathrm{rad}$. The sampling rate of the $\mathrm{A} / \mathrm{D}$ card was $200 \mathrm{kHz}$. Figure 5(a) shows the interference signal obtained by the A/D card. Figure 5(b) shows the frequency spectrum of the SMI signal. Figure 5(c) shows the demodulated phase. Figure 5(d) shows the retrieved displacement. The peak-to-peak amplitude of the reconstructed waveform in Fig. 5(d) was $12.168 \mu \mathrm{m}$.

Then, the PZT was controlled to move at the triangular wave form and trapezoid wave form with the frequency $10 \mathrm{~Hz}$ and amplitude $5 \mu \mathrm{m}$, respectively. Figure 6 shows the retrieved displacement. The peak-to-peak amplitude of the reconstructed waveform in Fig. 6(a) was $9.920 \mu \mathrm{m}$. The peak-to-peak amplitude of the reconstructed waveform in Fig. 6(b) was $10.140 \mu \mathrm{m}$.

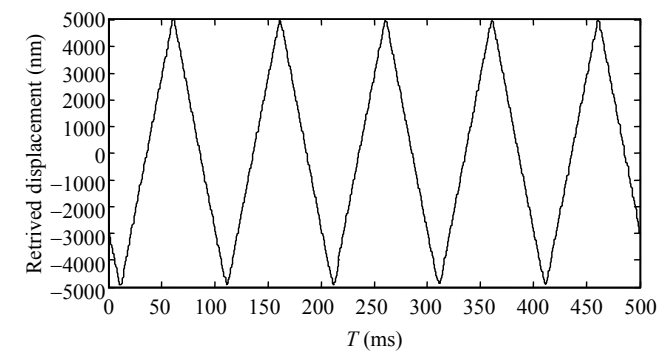

(a)

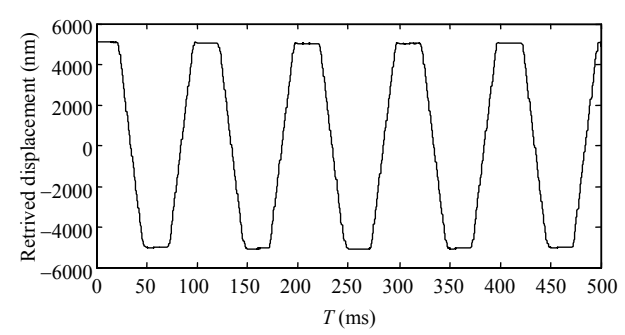

(b)

Fig. 6 Displacement measurement results with the frequency $10 \mathrm{~Hz}$ and amplitude $5000 \mathrm{~nm}$ : (a) triangular wave form and (b) trapezoid wave form.

In our experiment, the maximum measurement error was $84 \mathrm{~nm}$ with the $6-\mu \mathrm{m}$ measurement range. Thus, a measurement accuracy of $\lambda / 20$ could be obtained. The measurement precision has been improved compared with the previously reported fiber SMI techniques for displacement measurement $[12,13]$. A wider measurement range could be obtained with a higher modulation frequency and sampling frequency, and it needs the further work.

\section{Conclusions}

In this paper, a sinusoidal phase shifting self-mixing interferometer based on fiber laser for micro-displacement sensing has been developed and experimentally demonstrated. The measurement principle is described in detail, and the sources of the error influencing the measurement accuracy are discussed. In the experiments, the microdisplacement was reconstructed with the resolution $\lambda / 20$. The integrated-optic phase modulator used in the experiment made high-precision measurement available and easy to build a low-cost fiber sensing system. The system will have the potential for sensing applications, especially, in some places where laser can not be conveniently used in the free space for measurement.

\section{Acknowledgment}

This work was supported by the National Natural Science Foundation of China (Grant No. 91123015, 51405240), the Specialized Research Fund for the Doctoral Program of Higher Education (20113207110004), and the Natural Science Foundation of Jiangsu Province(BK20140925).

Open Access This article is distributed under the terms of the Creative Commons Attribution License which permits any use, distribution, and reproduction in any medium, provided the original author(s) and source are credited.

\section{References}

[1] H. N. Hansen, K. Carneiro, H. Haitjema, and L. D. Chiffre, "Dimensional micro and nano metrology," Cirp Annals - Manufacturing Technology, 2006, 55(2): 721-743.

[2] H. Martinussen, A. Aksnes, and H. E. Engan, "Wide frequency range measurements of absolute phase and amplitude of vibrations in micro- and nano-structures by optical interferometry," Optics Express, 2007, 
15(18): 11370-11384.

[3] L. Chassagne, M. Wakim, S. Xu1, S. Topçu, P. Ruaux, P. Juncar, et al., "A 2D nano-positioning system with sub-nanometric repeatability over the millimetre displacement range," Measurement Science and Technology, 2007, 18(11): 185-191.

[4] G. Li, Y. LI, K. Yang, and M. Liu, "Fiber-optic displacement sensor based on the DBR fiber laser," Photonic Sensors, 2014, 4(1): 43-47.

[5] B. Bodermann, E. Buhr, G. Ehret, F. Scholze, and M. Wurm, "Optical metrology of micro- and nano-structures at PTB: status and future developments," in Ninth International Symposium on Laser Metrology, Bellingham, October 3, 2008.

[6] W. M. Wang, W. J. O. Boyle, K. T. V. Grattan, and A. W. Palmer, "Self-mixing interference in a diode laser: experimental observations and theoretical analysis," Applied Optics, 1993, 32(9): 1551-1558.

[7] G. Giuliani, M. Norgia., S. Donati, and T. Bosch, "Laser diode self-mixing technique for sensing applications," Journal of Optics A: Pure and Applied Optics, 2002, 4(6): s283-s294.

[8] G. Giuliani, S. Bozzi-cietra, and S. Donati, "Self-mixing laser diode vibrometer," Measurement Science and Technology, 2003, 14(1): 24-32.

[9] D. Han, M. Wang, and J. Zhou, "Self-mixing speckle in an erbium-doped fiber ring laser and its application to velocity sensing," IEEE Photonics Technology Letters, 2007, 19(18): 1398-1400.

[10] L. Lu, Z. Cao, J. Dai, F. Xu, and B. Yu, "Self-mixing signal in $\mathrm{Er}^{3+}-\mathrm{Yb}^{3+}$ codoped distributed Bragg reflector fiber laser for remote sensing applications up to $20 \mathrm{~km}$," IEEE Photonics Technology Letters, 2012, 24(5): 392-394.
[11] X. Dai, M. Wang, Y. Zhao, and J. Zhou, "Self-mixing interference in fiber ring laser and its application for vibration measurement," Optics Express, 2009, 17(19): 16543-16548.

[12] Y. Zhao, M. Wang, J. Zhou, and X. Dai, "Self-mixing interference in fiber ring laser with parallel dual-channel," IEEE Photonics Technology Letters, 2009, 21(13): 863-865.

[13] H. Lu, M. Wang, X. Dai, and W. Guo, "All-fiber self-mixing interferometer based on DFB laser and phase modulating technique," IEEE Photonics Technology Letters, 2011, 23(4): 221-223.

[14] O. Sasaki and H. Okazaki, "Sinusoidal phase modulating interferometry for surface profile measurement," Applied Optics, 1986, 25(18): 3137-3140.

[15] D. Guo, M. Wang, and S. Tan, "Self-mixing interferometer based on sinusoidal phase modulating technique," Optics Express, 2005, 13(5): 1537-1543.

[16] L. Lu, J. Yang, Y. Zhao, Z. Du, and B. Yu, "Self-mixing interference in an all-fiberized configuration $\mathrm{Er}^{3+}-\mathrm{Yb}^{3+}$ codoped distributed Bragg reflector laser for vibration measurement," Current Applied Physics, 2012, 12(3): 659-662.

[17] X. Dai, M. Wang, Y. Zhao, and J. Zhou, "Self-mixing interference in fiber ring laser and its application for vibration measurement," Optics Express, 2009, 17(19): 16543-16548.

[18] O. Sasaki and H. Okazaki, "Analysis of measurement accuracy in sinusoidal phase modulating interferometry," Applied Optics, 1986, 25(18): 3152-3158.

[19] B. Ovryn and J. H. Andrews, "Phase-shifted laser feedback interferometry," Optics Letters, 1998, 23(14): 1078-1080. 